\title{
The complete denitrification pathway of Geobacillus thermoleovorans
}

\section{Duarte Molha*, Catherine Hack and Roger Marchant}

\author{
Address: Biomedical Sciences Department, University of Ulster, Northern Ireland, BT52 1SA, UK \\ Email: Duarte Molha* - molha-d1@ulster.ac.uk \\ * Corresponding author
}

from BioSysBio 2007: Systems Biology, Bioinformatics and Synthetic Biology

Manchester, UK. II-13 January 2007

Published: 8 May 2007

BMC Systems Biology 2007, I(SuppI I):P2 I doi: I0.I I86/I752-0509-I-SI-P2 I

This abstract is available from: http://www.biomedcentral.com/I752-0509/I?issue=S I

(c) 2007 Molha et al; licensee BioMed Central Ltd.

\section{Background}

Denitrification is a component of the global nitrogen cycle. It is an alternative process for respiration used by bacteria under low oxygen conditions, through which they can reduce nitrate and/or nitrite to dinitrogen gas through the production of the intermediates nitric oxide and nitrous oxide [1]. In recent studies, the thermophilic bacterium Geobacillus thermoleovorans was shown to reduce nitrate to nitrogen gas, indicating the presence of a denitrification pathway [2]. The first catalytic step of this pathway can be achieved by two alternative enzymes: a membrane-bound or periplasmic-bound reductase (NAR and NAP, respectively). The second step can be catalyzed by a CD1 nitrite reductase (nirS) or, in some species, a copper nitrite reductase (nirK). The third step catalyses the conversion of nitrite oxide to nitrous oxide by two different types of nitric oxide reductase. Finally the last step the catalization of nitrous oxide into dinitrogen gas completes the denitrification pathway and is carried out by the enzyme nosZ.

The aim of this study was to identify the key proteins involved in this pathway in the recently sequenced Geobacillus thermoleovorans genome, using standard bioinformatics tools.

\section{Materials and methods}

Data gathering and database creation

A database of all the published denitrification genes and other proteins associated with this pathway was assem- bled and used as probe sequences to search the genome assembly of Geobacillus thermoleovorans using BLASTP and BLASTX [3]. If no significant hits were found, the step was repeated using the non-redundant sequence database (nr) from the NCBI as the target and the BLASTP. BLASTX and PSI-BLAST [4] algorithms. All significant hits where collected and again used as probe sequences against our genome assembly. Finally all orthologous sequences were aligned and hmm profiles [6] were produced and searched against our genome assembly.

\section{Phylogenetic analysis}

The amino acid sequences of each denitrification gene were aligned using ClustalX [5]. A neighbor-joining tree was calculated for each using MEGA3 software $[7,8]$.

\section{Results and discussion}

In our genome of interest (Geobacillus thermoleovorans), we were able to identify most of the components of the first step on the denitrification pathway. We determined the presence of a narGHJI gene cluster and the narXL regulatory system as well as a possible nitrate/nitrite transporter homologous to narK gene. We also verified the presence of the periplasmic nitrate reductase napA although no other homologs of enzymes related to this alternative nitrate reductase were found. For the second step of the pathway, although no homologous for cd1-nitrite reductase were found, nirM, the electron donor for this enzyme is present together with nirE, an enzyme involved in the heme $\mathrm{d} 1$ bio-synthetic pathway. No other nitrite reduct- 
ase was found although a copper nitrite reductase has been identified in the genome of Geobacillus kaustophilus, a closely related organism. The third step of the pathway is represented by a homolog of the Ralstonia eutropha nitric oxide reductase. This is a dehydrogenase enzyme that accepts electrons from quinol. No enzymes where found related to the forth and final step of the pathway. However, in addition to the above proteins, several important regulatory proteins were also identified such as the fhp (flavohomeprotein) and the rpoN (sigma factor s54), $\mathrm{dnrN}$, norR and norQ that have important functions such as activation of different parts of the denitrification pathway depending on the availability of oxygen and/or nitrogen.

\section{Conclusion}

The main objective of this paper was to give an overview of the genes involved in the denitrification pathway of Geobacillus thermoleovorans. This was partially accomplished.

The first step of the denitrification pathway is well represented in this geobacillus species. We were able to identify the key genes that encode the enzymes required for the conversion of nitrate to nitrite by the nitrate reductase NAR. Although there are 2 alternative nitrate reductases identified on the genome, the periplasmic nitrate reductase does appear isolated with no other enzymes related to this membrane-bound nitrate reductase pathway. The enzyme responsible for the reduction of nitrite to nitrite to nitrite oxide was not identified although we have identified enzymes related to the bio-synthesis of the heme D1 required for the function of this reductase. For the third step of the denitrification pathway, we have identified an unusual nitric oxide reductase, present in just a few betaproteobacteria and, the related enzymesnecessary for the metabolic step were identified on the genome, although they are not clustered together in an operon has seen in Ralstonia eutropha. According to our genome assembly we could not identify any candidates for the last step of the denitrification process which indicates that this step might be absent in this organism. Unpublished laboratory results have indicated that this organism reduces nitrate and nitrite oxide; this implies that a nitrite reductase is present and we have failed to identify it. This may be due to errors in the draft genome assembly of G. thermoleovorans or that there might be an alternative enzyme responsible for the reduction of nitrite that does not share sequence similarity to the nitrite reductases identified until now.

\section{Acknowledgements}

The author would like to acknowledge the financial support by the European Union through its ESF grant to MSc study in Bioinformatics at the University of Ulster, UK [9].

\section{References}

I. Zumft WG: Cell biology and molecular basis of denitrification. Microbiol Mol Biol Rev 1997, 6 I (4):533-616.

2. Pavlostathis SG, Marchant R, Banat IM, Ternan NG, McMullan G: High growth rate and substrate exhaustion results in rapid cell death and lysis in the thermophilic bacterium Geobacillus thermoleovorans. Biotechnol Bioeng 2006, 95(I):84-95.

3. Altschul SF, Gish W, Miller W, Myers EW, Lipman DJ: Basic local alignment search tool. J Mol Biol 1990, 2I 5(3):403-4I0.

4. Altschul SF, Madden TL, Schaffer AA, Zhang J, Zhang Z, Miller W, Lipman DJ: Gapped BLAST and PSI-BLAST: a new generation of protein database search programs. Nucleic Acids Res 1997, 25(17):3389-3402.

5. Jeanmougin F, Thompson JD, Gouy M, Higgins DG, Gibson TJ: Multiple sequence alignment with Clustal $\mathbf{X}$. Trends Biochem Sci 1998, 23(10):403-405.

6. Baldi P, Chauvin Y, Hunkapiller T, McClure MA: Hidden Markov Models of biological primary sequence information. Proc Natl Acad Sci USA 1994, 9 I: 1059-1063.

7. Saitou N, Nei M: The neighbor-joining method: a new method for reconstructing phylogenetic trees. Mol Biol Evol 1987, 4(4):406-425.

8. Kumar S, Tamura K, Nei M: MEGA3: Integrated software for Molecular Evolutionary Genetics Analysis and sequence alignment. Brief Bioinform 2004, 5(2): 150-163.

9. Molha D: European Social Fund. University of Ulster: European Union; 2004.

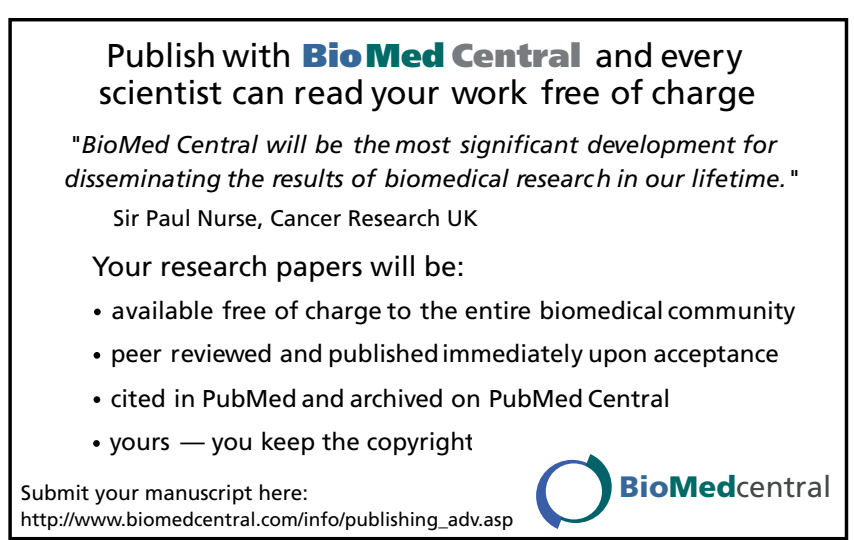

